\title{
Review of Control Methods and Strategies of Space Tether Satellites
}

\author{
Paul Tirop, Zhang Jingrui \\ Department of Dynamics and Control of Flying Vehicles, Beijing Institute of Technology, Beijing, China \\ Email address: \\ paultirop@yahoo.com (P. Tirop), zhangjingruibit@163.com (Zhang Jingrui)

\section{To cite this article:} \\ Paul Tirop, Zhang Jingrui. Review of Control Methods and Strategies of Space Tether Satellites. American Journal of Aerospace \\ Engineering. Vol. 4, No. 5, 2019, pp. 137-148. doi: 10.11648/j.ajtte.20190405.11
}

Received: March 27, 2019; Accepted: June 15, 2019; Published: December 4, 2019

\begin{abstract}
Space tether satellites systems are one of the most promising directions in the modern space industry. Such systems consist of two or more spacecraft connected to each other by very long tethers. Great extension and variable configuration of the system in the orbital flight conditions provide some dynamic features, which are not typical of conventional spacecraft. The concept of the tethered satellite system (TSS) promises to revolutionize many aspects of space exploration and exploitation. It provides not only numerous possible and valuable applications, but also challenging and interesting problems related to their dynamics, control, and physical implementation. The overarching theme of the paper is to show various control methods of the tethered satellites system (TSS) that have been undertaken recently, and also to emphasize on the importance of the TSS control method as an important aspect in the tether concepts, design, and missions. This review article presents the historical background and recent hot topics for the space tethers, and introduces the dynamics and control of TSSs in a progressive manner, from basic operating principles to the state-of-the-art achievements. The paper introduces the strategies and methods applied in controlling the TSS not excluding their advantages and disadvantages during the tether satellite deployment, retrieval, and station keeping procedures. At the end of the paper, a conclusion is made about the effectiveness of the control methods in stabilizing the libration and vibration motions of the TSS.
\end{abstract}

Keywords: Tether Satellites, Control Strategies, Control Methods

\section{Introduction}

The Tethered Satellite Systems have been in use for more than three decades now. Different concepts have been proposed on their application in space exploration. These concepts comprise of the generation of electricity, upper atmospheric research, and scientific experiments in the microgravity environment, cargo transfer and finally deep space observation. A lot of missions have been carried out to verify these concepts [1-3]. An example is a retrieval of the tethered satellite system (TSS-1) in 1992 and the deployment of a $20 \mathrm{~km}$ tether known SEDS-1 in space in1993. Also in the year 1994, closed-loop control of TSS (SED-2) during deployment was successfully performed $[4,5]$. The dynamics and control problem of tethered satellite systems has attracted wide attention for the past years as it is seen from the works of Decou, Kashmiri, Misra, and Farquhar [6-10]. The figure 1 below represents the forces due to gravity gradient at different heights.

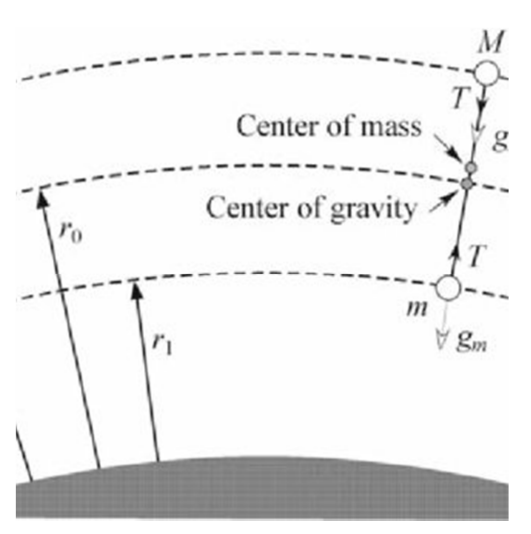

Figure 1. Forces due to the gravity gradient at different heights.

Marino and Tomei achieved a major breakthrough on adaptive output feedback control of a dynamical system. Their work prompted to increase in control development in the following years $[11,12]$ They were able to clarify that the 
nonlinearities in the output were not allowed to precede the input. In the companion paper, a system was considered not to be linear with respect to the unknown parameter vector [13]. Final results were obtained for set-point regulation problems. In the most recent paper, Marino and Tomei expounded on the results obtained in the previous papers for a class of systems with time - varying parameters [14].

Krsti'c and Kokotovi'c in their paper advanced the control approaches developed by Marino and Tomei. They used such concepts as "tuning functions" and "swapping-based" schemes to allow for the standard gradient update law to deal with the parameterization [15]. A paper by Khalil in the other hand explained about the adaptive output-feedback control of single-input-single-output systems which can nonlinearly be dependent on the control input [16].

The concept of interconnecting spacecraft by using lightweight deployable tethers has also been important for space observations. This is because of the high levels of propellant consumption demanded by the separated spacecraft information. This can be significantly reduced by controlling the tension or length of the interconnecting tethers [17-20].

This paper is organized as follows. It begins with a discussion of the control strategies of the TSS models as applied in some relevant papers of research. The next section presents a detailed description of the control methods used in the deployment, retrieval and orbit maneuver of the TSS in reference to the relevant published research papers as per the operation period of the TSS to date. Finally, the conclusion and recommendations are made in relation to future work on the control methods of TSS.

\section{Control Strategies of the TSS}

One of the interesting control applications for tethers is based on the dynamic isolation of payloads from the main satellite. This concept has been fully discussed in the paper written by Ohkami et al. [21]. The authors describe a three mass system model that is connected in series by two tethers. The mass system comprises of the base vehicle, the platform, and ballast mass. The rotations and the translations of the platform are taken into account and their equations linearized. One of the assumptions was related to the state of equilibrium of the mass system after a deflection that was meant to be small. For simple feedback control, a very highly accurate microgravity manipulation was available. According to Licata, it is necessary to concentrate on the nonlinear dynamics especially when handling cases on waste disposal and capsule re-entry for a largely controlled motion [22]. Therefore alternatives needed to be employed such as fuzzy logic which could provide very effective control to the TSS.

Nohmi et al. investigated the control of robots remote from their space vehicle [23]. The work could also be referred to [24] where it was revisited again. In [24], the translational momentum on the center mass of the tethered robot is controlled by applying the tension force with the tether in respect to its robot's mass center. Manipulations of tether tension and link motion are seen to affect the control of robotic motions on the space vehicle. The reactions from the wheel, jet, or thrust, are required to control angular momentum about the tether. In the paper, it is shown that the link motion of the tethered robot could be split into two reliable sub-tasks, a) end-effector motion and b) tether attachment motion. The compensations from the impulsive disturbances are found to be robust and effective.

The paper by Kumar and Kumar discusses the control of system motion on two spacecraft that are connected together by the use of four tethers [25]. The tethers are subjected to tensional moments. The authors selected a control law known as the combined- open - loop and a simple feedback scheme for controlling the motion of the system. They considered two control motions, namely the longitudinal system drift and attitude excitation. They assumed that the TSS comprised of two identical satellites connected with short tethers with their anchor points located on the principal roll axis and are symmetrically offset from the center mass of each satellite. The mass of the tether was neglected and planar angular motion case was considered. The Pitch angles on the two satellites, tethers, and the length of the tether expressed the motions of interest and comprised of three angles and one translational coordinate. From the paper, the effective control was achieved using tethers as short as $10 \mathrm{~m}$. From the writers' point of view, the combination of open-loop and feedback control provided an improvement in attitude precision for system alignment along with the line-of-sight. It was proposed as the most viable alternative of maintaining the station maneuvers for geostationary satellites, especially when onboard fuel is next to exhaustion.

Pradhan et al. in his paper investigated on the offset control of a tethered sub-satellite from a large platform. In the paper, offset mechanism assumes the form of a manipulator attached to the platform and is capable of providing movement to the platform end of the tether in the local horizontal and vertical directions [26]. Pradhan et al modeled the tether to appear as a flexible string and applied the assumed modes method for discretization. The system motions are defined by the tether modal coordinates, generalized coordinates, and the orbital plane. Damping was included by use of Rayleigh dissipation function. The generalized force vector represented the momentum gyros located near the center mass of the platform and thrusters at the end of the tether sub-satellite. Modeling accuracy was done by checking on the total system energy and comparing the frequencies in the linearised system with those available in the literature. Feedback Linearization Technique (FLT) was used for controlling the attitude dynamics whereas a robust LQG was used for controlling the vibrational modes. Results of the overall offset control were effective for the regulation of platform pitch and tether vibrations but not effective on the tether attitude, where large offset motions were needed.

A very good means of testing the microgravity effects with the assurance of a fair deal in controllability was investigated in a paper by Fujii et al. [27]. A three degree of freedom 
nonlinear analytical model with a floating balloon at its upper end and ready for terrestrial deployment was considered. A control law was designed to control the motion of the system and to adjust the length of the tether. The tension provided by the balloon was indicated in Newton. The experiment and simulations of the analytical model are compared and the concept of virtual mass is combined with the equations of motions of the analytical body. It was found that the fluid surrounding the accelerating body inside the balloon seemed to increase the mass of the body. From the results, the presence of virtual mass contributed to the improvement in the accuracy of the model.

A paper by Kumar and Yasaka explains the attitude stabilization and configuration -based control of tethered spacecraft [28]. In this work, the authors demonstrated how a single tether that connects the main satellite or vehicle to an auxiliary mass required a feedback control to stabilize the attitude of the satellite. When the two-tether system was engaged, an improvement on the system performance was achieved. The authors presented a model form of a kite consisting of three tether spans. The first two spans came from the points on the center mass of the (upper) satellite symmetrical offset and ended on the common connecting point where the third span was hanging down. The authors gave a summary on the whole concept using a nonlinear, non-dimensional, Lagrangian model that consisted of 10 general coordinates. The results showed that the stability analysis performed for the linearized system about equilibrium possessed certain physical constraints that were necessary for stability which could be potentially achieved [29].

Authors Cho and McClamroch discussed the stabilization and control of single-tethers in their work [30]. The control objective in their case was slightly stricter; it required not only attitude control of the satellite, but also the consistency of the small tether motions. They applied two ways; the first one being the application of decoupling method to attitude dynamics from the tether dynamics, and second way was by using a Kalman decomposition to decouple the uncontrollable modes and then stabilize them by using the linear feedback method. The conclusion derived stated that the Kalman decomposition approach was best for the rollyaw attitude stabilization which is more demanding as compared to the pitch control, because of the less dynamic and actuator movement requirement.

Pela' ez and Lara researched on the instability and control of EDT. Instability is independent of tether flexibility and so the tether is modeled as a dumb-bell with end masses [31]. The Geomagnetic field is represented by a non-tilted dipole model. The tether current is assumed to be constant. The electrodynamic force and the system dynamics equations on the paper provides a detail explanation of the stability of the tether in relation to the orbit inclination and the parameters that represent the magnitude of the ED force on the tether. Previous asymptotic analysis is extended by the use of numerical algorithms based on the Poincare' method on the continuation of periodic orbits. The high inclination is not initially seen to be appropriate for vertical ED tether as shown from the paper because for a given inclination there is the critical value of the ED magnitude parameter. In such a case it is the current control of the tether which could help to alleviate such effects, but from the authors' recommendations, such tethers are supposed to be operated away from this type of threshold. Authors also show that there are many unstable periodic solutions for this type of tether system and that such regimes are unsuitable in longterm operations of the EDT.S.

Rossi et al. in a 2004 paper provided an interesting account of a likely periodic motion of a tether trailing satellite, with the attention being paid to both the motion of the satellite and the tether [32]. The paper illustrated a situation whereby a tether connected to two satellites was cut as a result of an accident or a planned maneuver. Several assumptions were made, for example, it was assumed that the earth centeredframe was inertial, the satellite could be a point mass, the tether was homogeneous and comprised of uniform density, the torsional and transversal vibrations on the tether could be neglected, and elasticity followed the Hooks law. The model comprised of partial and ordinary differential equations. The work showed that the existence of periodic solutions for such a system did not depend on the equilibrium state when gravitational and oblateness terms predominantly drove the dynamics. The important features related solely to tether density, length, flexibility, and rotational speed. The shorter systems exhibited periodic motions about their equilibrium states. The tether trailing satellites were strongly influenced by the equilibrium state when the atmospheric drag influenced the system. The reason was that the gravitational and oblateness forces were uniformly bounded independent of position as compared to the drag forces. The existence of periodic motions with bounded forces was found to depend just on tether parameters whereas the unbounded (linear) growth depended on the equilibrium states [33 - 38]. Figure 2 below shows how forces can be restored on tethered end bodies.

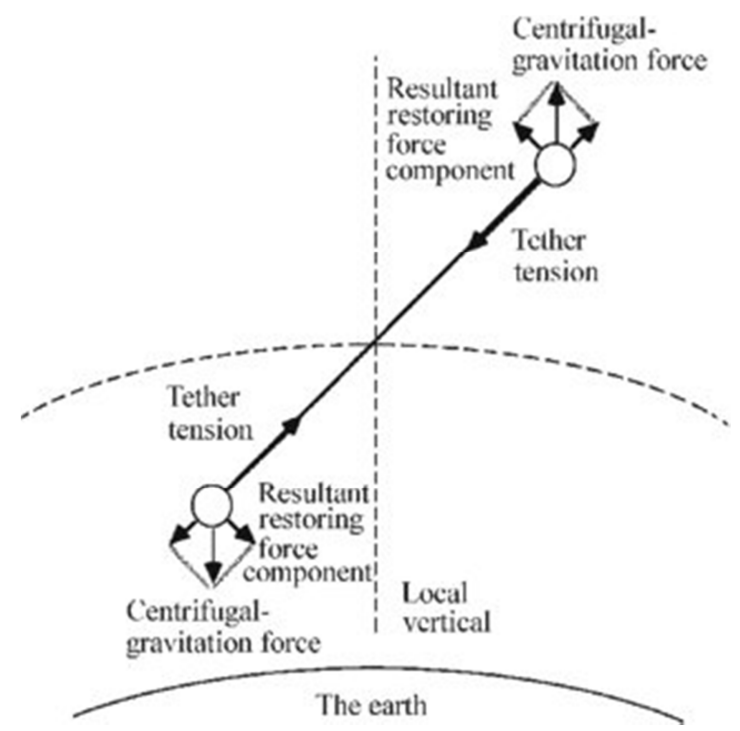

Figure 2. Restoring forces on tethered end-bodies. 


\section{Control Methods of the TSS}

\subsection{Open-loop Control Method}

Bombardelli et al. studied the attitude dynamics of a linear tethered formation based on perturbation methods and applied an open-loop control strategy for efficiently changing the plane of rotation of the formation by using a pair of electric thrusters in an optimized way [38].

In [39], a simple open-loop tether offset control law for attitude maneuver of dual satellite platforms connected by a relatively short tether was proposed. However, the system had the limitation of controlling yaw excitation in the case of roll maneuver.

\subsection{Finite Thrust Control Method}

Beard and Hadaegh proposed this method in their paper. The method was used for a satellite formation rotating at a constant rate in free space [40].. The disadvantages of the method are the reduction of thruster fuel and the decline in position control accuracy. That is why a coordinated control method was formulated to save thruster fuel and improve control accuracy.

\subsection{Coordinated Control Method of Tethered Satellite}

The paper by Osami Mori and Saburo Matunaga best describes the application of this method [41]. It proposes a new tethered satellite cluster system as shown in Figure 1.

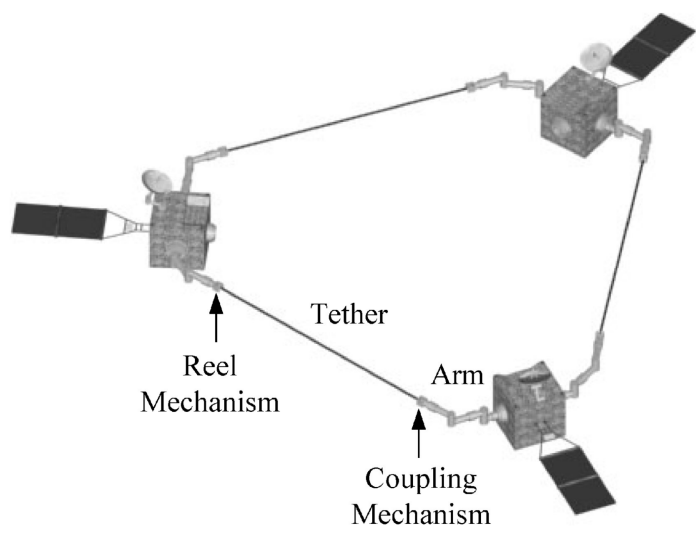

Figure 3. Tethered satellite cluster system.

From the diagram illustrated in Figure 3, the system is seen to have three tethers that are connected to robotic satellites models. The robotic model maintains and changes formation using active control of the tension and length of each tether. The satellite is installed with arms which can change the tether offsets by controlling their endpoints. This implies that the tether tension can not only be used for saving thruster fuel but also for improving the control accuracy of attitude and position of the satellites in the cluster system [41-44]. The above concept can be used in tethered multi satellites systems for in-orbit servicing by engaging in several missions, for example, recovery, casting, inspection and deorbiting of satellites that are not controlled [45-48].

The paper also considers the rotational motion with respect to the center mass on the system. The circular motion is treated as the most essential item. The equilibrium conditions for maintaining a circular motion using the tether tension are displayed, and the coordinated control method based on the equilibrium conditions in minimizing thruster fuel is investigated. Afterward, the equilibrium conditions for the same transformed system are derived and methods of fuel control are displayed. The results show that the coordinated control methods (for circular and non-circular motion), are effective in reduction on fuel consumption and attitude errors in the satellites.

\subsection{Nonlinear Adaptive Control Method}

Research papers authored by DeCou [6, 7], Keshmiri and Misra [8], Wong and Misra [49] and Farquhar [10] demonstrates the use of nonlinear adaptive control in the dynamics and control of the tether satellites. The paper by Kim and Hall provides a thorough review of the nonlinear adaptive control of dynamical systems which of recent has emerged as an important approach to nonlinear controller design [11]. Marino and Tomei made the most important breakthrough on the adaptive output-feedback control [12, 13]. Their work led to a remarkable development in the following years. The authors managed to remove the structural restriction on the nonlinearities in the output because it was not allowed to precede the input. In the companion paper they considered a more general class of systems whereby the system was not required to be linear in respect to the unknown parameter vector. In the recent paper, Marino and Tomei extended the results obtained from their previous paper [12] for a class of systems with time-varying parameters [14].

Krstić and Kokotović advanced the control approach that was previously developed by Marino and Tomei [15]. They introduced new scheme concepts based on "tuning functions" and "swapping-based" that could allow the inclusion of standard gradient update law. Khalil considered the problem of adaptive output-feedback control on single-input-singleoutput systems, which could be nonlinearly dependent on the control input [16]. From the research, he could only achieve semi-global stability of the tether. Moreover, knowledge of bounds on parameters and initial conditions were a requirement. The persistence of system excitation was sufficient on both the parameter and tracking error convergence. Lora presented the first paper on global outputfeedback control for one-degree-of freedom with the EulerLagrange systems [50]. The control design exploited the properties of hyperbolic functions so as to define a "nonlinear approximate differentiation filter" that could automatically enlarge the domain of attraction. The paper by De Queiroz et al. used a similar approach to Loris' to achieve global stability for general $n$ th-order of uncertain systems [51].

The most recent paper by Mischa Kim and Christopher D. Hall demonstrates the use of nonlinear adaptive control technique in dynamics and control of rotating tethered satellite systems [1]. Comparison is done between two 
tethered satellite configurations that is a triangular system and more complex formation referred to as Tetra-Star [18]. The Tetra-Star system consists of three controlled spacecraft and three uncontrolled counterweights. This is shown in Figures 4 below.

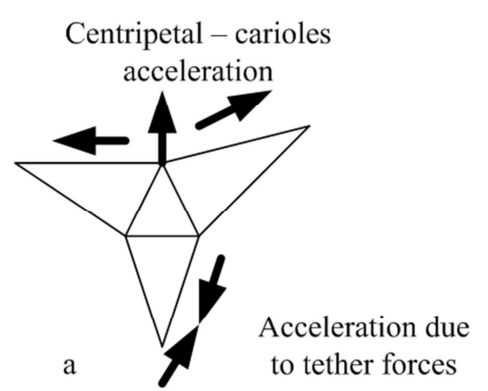

to tether forces

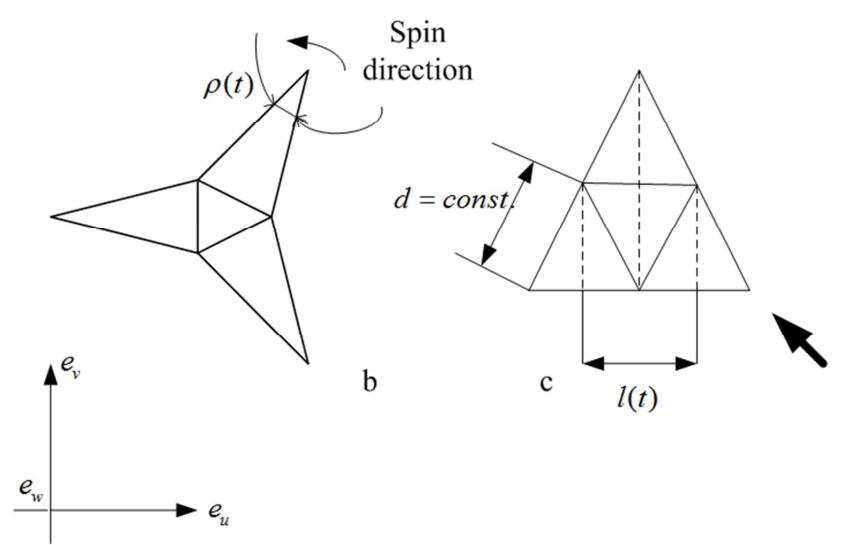

Figure 4. Schematic layout of Tetra-Star during deployment tether.

The inner point masses represent the controlled spacecraft which forms the "inner" system. The outer system or outer point masses represents the uncontrolled counterweights. The three tethers interconnecting the controllable spacecraft are deployable while the unstrained tether length $d$ remains constant. It is noted that the triangular configuration could be obtained from the Tetra-Star model by the removal of the outer point masses and the corresponding tethers. Tetra-Star offers major benefits as compared to the triangular TSS in relation to its control technique. The Tetra-Star model control performance is strictly based on its contribution to the total control impulse in the angular direction and regulation of the angular momentum of the TSS according to the desired trajectory. It should also provide authority to the radial control component which contributes to the overall control effort only when the TSS is in the transitional phase between a steady spin and deployment or retrieval. This is represented in Fig. 1a where the additional force components are identified by the use of full arrows whereas the centripetalCoriolis acceleration component is identified by using the broken arrows. Lastly, if the counterweights were to be dimensioned correctly, then they would act as a buffer to balance the increase and decrease in angular momentum during tether deployment and retrieval.

The advantage of the triangular system is its superiority in terms of parameter estimation as compared to the Tetra-Star. In Tetra-Star there is a requirement not to control the outer spacecraft which at the end renders the satellite formation to be unstable and uncontrollable. From the control point of view, the Tetra-Star portrays more benefit. The control in a radial direction is negligible, and in angular direction there is a decrease in overall control impulse. The overall effort is reduced by allowing the outer tethers to be deployable by using a deployment retrieval strategy that will render the angular momentum of the tethered satellite system constant. The disadvantage of the Tetra Star is the lack of its control authority with the uncontrolled counterweights turns out to be irrelevant for in-plane maneuvers, therefore, affecting the retargeting maneuvers.

\subsection{Decoupling Control Method/ Model-following-Decoupling Control Method}

Decoupling control method has been put into application in the paper written by Isidori A. According to the paper, the decoupling control method can be used to control each tether attitude independently [52]. The independent motion is suitable for achieving satellite constellations effectively and various scientific missions will be possible with the application of the independent motion for a three mass tethered satellite system. These will include the observation of planets with a magnetic field and the aurora from more than two directions simultaneously.

Another paper by Kojima, Iwasaki, Fujii, Blanksby, and Trivailo also expounds on the decoupling control method and the modell -following- decoupling method [53]. The control method is introduced for the purpose of achieving a periodic motion that can study the earth atmosphere at a specific altitude periodically. The periodic in-plane motion of a tethered satellite system in a circular orbit is considered as the reference trajectory. It is used for tracking by the actual tethered satellite system in an elliptic orbit.

Kojima and Sugimoto employed the decoupling and model-following-decoupling methods to perform a nonlinear control of a double pendulum in electrodynamic tethers systems (EDT) [54]. They used nonlinear control of liberation motion to find the nonlinear control input where each output could be controlled independently. The authors applied the Morgan problem to equalize the dimensions of the output and that of the control input. This transformation of obtaining a nonlinear control input was then referred to as the decoupling method. The method was then used by the authors to control the angles of the EDT system independently. The model-following-decoupling control method was initially used to specify the reference model that behaved desirably. It was later used to control the response of a plant that converged asymptotically to the reference model by providing the plant with feedback control inputs based on 
the differences between responses. The results showed that the chaotic libration motion of each angle on the electrodynamic tethers could be stabilized independently using the decoupling control method. Model-followingdecoupling control method possessed good performance in tracking the trajectory of the reference system. This was shown by its application on the electrodynamic tethered system.

\subsection{Nodal Position Finite Element Method/Current Switching Method}

According to the paper by Corsi and Less the nodal position finite element method can be used for stability control of liberation motion of the EDT [55]. In the case of the absence of control devices e.g. thrusters, the only controllable parameter for the stability becomes the electrical current. At this juncture, a specific Lyapunov function is defined as stability function to control the libration motion, where the threshold energy level of the libration motion was meant to control the on and off switching. Results showed that the amplitude of libration could be bounded within a presented range by a control strategy in the deorbiting process.

Tortora et al proved that a microsatellite could be easily and efficiently deorbited [56]. This could be done without the loss of stability of the EDT by applying the Lyapunov function and libration angle to angle rate.

A recent paper by Sun, Zhu and La Rosa proved the importance of nodal position finite element method in dynamic modeling of a cable towed body [57]. The method according to them can be able to handle a rigid-body motion coupled with small elastic deformation in a simple and accurate manner. The perturbation forces in the orbit that the EDT may experience in low earth level (LEO) include, a) earth atmospheric drag, b) thermal functions of the tethers, c) solar radiations, d) lunisolar gravitational force, e) the electrical dynamic force on current- carrying tethers and $\mathrm{f}$ ) Non homogeneous gravity field.

A paper by Ganqiang Li developed two practical and effective electrical current with on and off control strategies on the basis of libration energy and librational angles. It is found out that the libration energy control is more stable and efficient [58]. The libration angle is more on practical applications because of its simplicity and low computational effort. The paper concentrates on the bare EDT system. The bare EDT system was seen to portray a big advantage in terms of active space debris removal (ASDR) because it appears simple in nature, has high efficiency in collecting the current, it is reliable in operation and it is flexible to be adopted by different spacecraft.

\subsection{Propellant-less Technology}

This method can be used to provide both attitude and orbital control without limiting the lifetime of the spacecraft $[59,60]$. This method is dominantly applied to "chipsats" for controlling them with multiple electrodynamic tethers.
The paper authored by Lorraine and Mason researches on this particular method. In the paper, a multiple electrodynamic tether propulsion system is used to provide rigidity to the tethers by shortening them as compared to the larger spacecraft where additional structures are required to keep the tethers in a rigid position [61]. Specific configurations of multiple tethers can guarantee control in any direction or orientation allowing simpler and more agile control than on the traditional single EDT. In a multiple EDT system, each tether can be independently controlled by its own current and direction. Multiple EDT possesses the following advantages, 1) there are multiple directions of action available at a single time. This provides more precise control and enables to eliminate some of the controller dependence on the orbital geometry, 2) they also provide flexible propellant-less propulsion for different variety of missions, therefore larger systems can benefit from the method of propellant-less for attitude control and orbit stabilization.

\subsection{Sliding Mode Control Method}

Sliding mode control method (SMC) is one of the prominent robust nonlinear control methods used in solving issues related to uncertainties and disturbances. It is also a variable structure control method because of its statefeedback control law that is not a continuous function of time. This means that it can move from one continuous structure to another based on the current position in space. The SMC also alters the dynamics of a nonlinear system by use of a discontinuous control signal. The signal forces the system to "slide" along a cross-section of the system's normal behavior. The multiple control structures are designed to enable the trajectories to move toward a neighboring region with a dissimilar control structure. This will enable the ultimate trajectory not to exist entirely within one control structure. Instead, it will slide along the boundaries of the control structures. This motion of the system is therefore referred to as the sliding mode and the geometrical locus to the boundaries is called the sliding (hyper) surface.

A paper by S. V. Drakunov and V. I. Utkin explains the concept of sliding modes in abstract dynamic systems described by the introduction of a semigroup of state space transformations [62]. The sliding mode design procedure is used for designing finite observers, sliding mode control for systems with delays and differential-difference systems of which all are illustrated by sliding mode control of longitudinal oscillation.

The sliding control methodology introduced by Slotine and Sastry (1983) was about the aspect of achieving an accurate tracking for non-linear time-varying multivariate systems in the presence of parameter variations and disturbances [63]. An explicit trade-off is obtained between tracking precision and robustness to modeling uncertainty: tracking accuracy is sot according to the extent, of parametric uncertainty and the frequency range of unmodell dynamics. The trade-off is further refined to account for time-dependence of model uncertainty. 
The paper by Mohamed A. A. H. and Nima Assadian explains the control of electromagnetic tethered satellite formation actuated by electromagnetic dipoles and reaction wheels using the robust sliding mode control technique [64]. Generating electromagnetic forces and moments by electric current coils provides an attractive control actuation alternative for tethered satellite system due to the advantages of no propellant consumption and no obligatory rotational motion. Equations of motions are derived based on the dumbbell model of tethered satellite in which the flexibility and mass of the tether is neglected. In the model, the perturbation is considered. The end and mid-field models of electromagnetic forces and moments of two satellites on each other and the effect of the Earth's magnetic field are presented. A robust sliding mode controller is designed for tracking the precise trajectory, and to also counter the e electromagnetic force and moment uncertainties and external disturbances as a result of the Earth's gravitational and magnetic fields inaccuracy. The end simulation results presented, proves the effectiveness of the developed controller and its superiority over the linear controller.

\subsubsection{Adaptive Sliding Mode Control Method}

Adaptive control deals with situations in which some of the parameters are unknown or time-varying. The main concept involves the estimation of these unknown parameters online and then applying them in place of the unknown parameters in the feedback control law.

The paper by Walls and Greene presented an adaptive control technique on an orbiting single tethered satellite system. The performance was rated based on the effectiveness of the controller in performing station-keeping maneuvers [65].

The paper by G. Bartolini, A. Levant, and E. Usai illustrates an adaptive second-order sliding mode control (2SMC) scheme which provides the estimates and compensations for the uncertainties affecting the system dynamics [66]. The 2-SMC adjusts the discontinuous control effort so as to reduce it to arbitrarily small values.

A paper by C. Edwards and Y. Shtessel proposes a supertwisting-like structure with adaptive gains. The structure constitutes of two parameterized scalar gains, both of which adopt by an additional time-varying term. The adapted terms are allowed to increase and decrease as appropriate in accordance with their magnitude so that they appear as small as possible and large enough to sustain a sliding motion [67].

In the paper by A. Barth, M. Reichhartinger, K. Wulff, M. Horn, and J. Reger a Lyapunov-based control concept is presented that combine variable structure and adaptive control [68]. The considered system class consists of nonlinear single input systems which are affected by matched structured and unstructured uncertainties. Resorting to the certainty equivalence principle, the controller exploits advantages of both the sliding-mode and the adaptive control methodology. It is demonstrated that the gains of the discontinuous control action may be reduced remarkably when compared with pure sliding-mode-based approaches.
The paper by Qinglei Hu presents a dual-stage control system design method for flexible spacecraft attitude maneuvering control by use of on-off thrusters and active vibration suppression by embedded smart material as actuator [69]. An adaptive sliding mode controller with the assumption of the upper bounds of the lumped perturbation is designed which ensures uniform ultimate boundedness (UUB) of the attitude control system in the presence of a bounded parameter. The adaptive controller is redesigned in such a way that the need for knowing the upper bound in advance is eliminated. By applying Lyapunov analysis, the modified adaptive controller guarantees the UUB of the system. In suppressing the induced vibration, linear quadratic regulator (LQR) control method is presented. Results show that rotational maneuver and vibration suppression are achieved in spite of the parameter uncertainty and saturation input. This was also done by Boskovic J. D, Li S. M and Mehra R. K [70].

According to a paper by Ma Zhiqiang and Sun Guanghui they proposed a novel adaptive sliding mode tension control method for deployment of the tether satellite. The input tension limitation in this paper is taken into account [71]. First, the governing equations of the tethered satellite system are derived using Lagrangian theory. The tether is considered to resist axial stretching and the tension input is modeled as input limitation. New adaptive sliding laws are used to provide the stability of the tethered satellite. Compared to the classic control strategy the newly proposed control law could deploy the satellite with smaller overshoot on the in-plane angle and implement on the tension control effectively.

\subsubsection{Fractional Order Sliding Mode Control Method (FOSMC)}

The FOSMC was developed for the purpose of sustaining tether deployment by considering the uncertainties. On its first application in space, it dealt with the attitude control problems of flexible spacecraft as explained in the paper by Manabe [72]. Several other fractional orders were then proposed by Khalil and applied to control the attitude of the satellite in the purpose of achieving a compromise between stability and performance [16]. It is an extension of the work done by Sun and Zhu where they combined both fractional order (FO) and SMC so as to arrive at the efficient control laws that could deploy the space tether in a stable, precise and fast way with the presence of environmental perturbations and uncertainties of the model [73]. The effectiveness of FOSMC is done by comparing it to the proportional-derivative method (PD), the fractional order proportion derivative (FOPD) and the standard integral order sliding mode (SIOSM) [74]. The results show that the SIOSM and FOSMC control methods do have more advantage than PD and FOPD in suppressing disturbances to the tether satellite system and in convergence in time. Also due to the fractional order derivative, the oscillations in FOPD appears to be less severe as to the PD oscillations. The reason is the FOPD tends to exhibit the characteristics similar to that of a filter. 
Advantages of FOSMC are: 1) it has a faster response with minimum time and small pitch angle as compared to SOSM; 2) it has a better performance as compared to the PD, FOPD and SIOSM [75].

\subsubsection{Terminal Sliding Mode Control Method}

Terminal sliding modes (TSM) was invented in the early 1990s (JPL) by Venkataraman and Gulati. It is evoked by the concept of terminal attractors which guarantee finite time convergence of the states [76]. In normal sliding mode, asymptotic stability is achieved which leads to the convergence of the states to the origin. The convergence at normal sliding mode may only be guaranteed within infinite time. In TSM, a nonlinear term is introduced in the sliding surface design so that the manifold is formulated as an attractor. After the sliding surface is intercepted, the trajectory is attracted within the manifold and converges to the origin following a power rule. The terminal sliding mode also has been widely applied to nonlinear process control, for example, rigid robot control in the tethered satellite. The terminal sliding mode was developed to provide finite time, stability and high accuracy when the system is facing strong disturbances. This can be seen from a paper written by Yang and Yang [77].

Table 1. Types of control sliding mode methods and their applications.

\begin{tabular}{ll}
\hline $\begin{array}{l}\text { TYPE OF SLIDING MODE } \\
\text { CONTROL METHOD }\end{array}$ & APPLICATION \\
\hline Adaptive sliding mode & Station keeping maneuvers \\
Fractional order sliding mode & Deployment, Retrieval, Maneuvers \\
(FOSMC) & Deployment, Retrieval, Maneuvers \\
\hline
\end{tabular}

\subsection{Feedback Linearization Control Method}

The paper by Pradhan et al. describes the tethered satellite system attitude and vibration control by use of a feedback linearization technique and a robust linear-quadraticGaussian/loop transfer recovery [26]. The results from the analysis performed, indicated that the tether offset scheme was effective in the simultaneous control of the platform and the tether pitch motion for a shorter tether.

The paper by $\mathrm{Yu}$, Jin, and Wen presents asymptotic stabilization strategy for the deployment of controlled tethered satellite system in three -dimensional space, where the tether length is taken as the control variable [78]. In the beginning, a rigid -rod tethered model is used to form the nonlinear dynamic equations of in-plane and the out-of-plane motions of the system. Later through the application of the feedback linearization control technique, the stability of the linearized system at deployment is obtained. The large swing motions during deployment are stabilized by the use of electrodynamic force and the tether length rate. The case studies in the paper well demonstrated the proposed stabilization control strategy.

\subsection{Delayed Feedback Control Method}

Kojima et al. proposed to control the librational motion of a three-mass TSS in an elliptic orbit by using the existing delayed feedback control [53], and designed an innovative control scheme by combining the delayed feedback control with model-following and decoupling-control method, to improve the control performance. In the next paper, Kojima with Sugimoto used the feedback delayed method to demonstrate the stability of in-plane and out-plane periodic motions of EDT in an inclined elliptic orbit [79].

Palaez and Lorenzini [80] introduced a delayed feedback control method known as time-delayed auto- synchronization of the system (TDAS) in their work. From their study, they showed that the TDAS control method failed to stabilize the basic periodic motions of the tether. The TDAS control method was not able to convert unstable periodic motions of the uncontrolled tether into stable ones for a short period of time. Another control method was then introduced to improve on the TDAS. The method was named, extension time delay auto-synchronization of the system (ETDAS) method which was introduced by Inarrea and Palaez In this method, a control variable was progressively delayed at the output for a specific limited time as compared to the TDAS where the control variable of the system was delayed at the output by amount of time [81]. The delayed control values were then reintroduced into the system through the feedback control signal. The control signal possessed two adjustable parameters the, 1) feedback gain and 2) memory parameter. When ETDAS was applied to the periodic motion, the delay time coincides with the period of the motion. The advantage of ETDAS as compared to TDAS is that it uses the information on the previous state of the system so as to stabilize the periodic orbit with the time period. From this concept, ETDAS had been successful in converting the unstable periodic motions to stable ones in the EDT lying on the inclined orbit.

A recent paper by Lanchares et al entails the study of two delayed feedback control methods (TDAS and ETDAS) combined together [82]. The results from the two methods showed that they were both able to transform the uncontrolled unstable periodic motions into stable ones. The advantage of the delayed feedback method is that it requires smaller control forces than the non-feedback technique in order to control the system.

\section{Conclusion}

\subsection{Optimal Control Method}

Williams applied these methods to the deployment and retrieval of a three-mass tethered formation spinning in the orbital plane [83]. He treated the slow orbital and fast liberation motions separately in the optimal control of the electrodynamic tether orbital transfer [84]. He employed the use of direct transcription methods to find the optimal trajectories for three kinds of tension-controlled maneuvers, which include minimum time reorientation, deployment, and retrieval. A modal analysis of these formations was also applied by linearizing the dynamic equations of lumped mass 
models. The optimal method has been applied in a tethered tug system where by the system is used for space debris removal. Although it is promising, the orbit experiments of space debris removal are still limited because of issues such as the tether slack, tether winding with tug and debris and also collisions between tug and debris during the orbital maneuver $[85,86]$. The purpose of optimal control is to stabilize the tethered space tug system in orbital transfer with less time and minimum fuel consumption when controlling the acceleration length of the tether. Current authors [87] have developed a timescale separation scheme that can optimize fast nanosatellite deorbiting by using a shorter electrodynamic tether, therefore, simplifying the optimal control method significantly.

\subsection{A Nonlinear Optimal Control Method}

This method was used to determine the periodic trajectories, as well as reconfiguration and retrieval trajectories for the complex double-pyramid configuration, whereas receding horizon control was applied to stabilize the station-keeping mode of the Earth-pointing formation in a circular orbit [88]. It is derived to control the attitude of a satellite using the tether offset variations. Advantage of this method is that the pitch motion of the TSS is stabilized back to its equilibrium position. It also performs better because of its small unstretched tether lengths. This is evident in the case of nanosatellites and picosatellites [89, 90]. The nonlinear optimal control method can be used to control TSS in case of tether failure by use of offset controls. This makes the concept more useful and attractive for future space applications.

\subsection{Summary}

This paper has attempted to provide the reader with a reasonably broad background on the field of space tether control research. Still, with considerable contributions made to the literature in recent years, control strategies and methods of the TSS remains an extremely active and vibrant research area for the TSS. The intention has been to provide the motivated reader with a significant reference resource, notwithstanding the very large number of papers and manuscripts that have been published. The review paper covers the control methods as applied to all types of TSS in general and it sets future agendas relating to the development of the control methods of the tethered satellites. From the review, the control strategies of the TSS have been analyzed based on the dynamic isolation of payloads from the main satellite. The concept has been fully presented by outlining and comparing different authors views on the control methods and strategies of TSS. Different types of control methods and strategies have been discussed based on the applications of the TSS. From the research, it can be concluded that the application of the control methods and strategies on the TSS depends entirely on the stages of operation of the TSS i.e. deployment, maneuvering, station keeping, and retrieval. It is also concluded that the control methods either separately or together can provide stability to the TSS during its space missions.

\section{References}

[1] Johnson, L., Glichrist, B., Estes, R. D., and Lorenzini, E., "Overview of Future NASA Tether Applications," Advances in Space Research, Vol. 24, No. 4, 1999, pp. 1055-1063.

[2] Maccone, C., "Tethered System to Get Magnified Radio Pictures of the Galactic Center From a Distance of 550 AU," Acta Astronautica, Vol. 45, No. 2, 1999, pp. 109-114.

[3] Quadrelli, M. B., Hadaegh, F. Y., Lorenzini, E. C., and Bombardelli, C., "Precision Tethered Formations for LEO and Space Interferometry Applications," December 2001, 16th International Symposium on Space Flight Dynamics, Pasadena, California.

[4] Cosmo, M. and Lorenzini, E., Tethers in Space Handbook, Cambridge, Massachusetts, 3rd ed., 1997, prepared for NASA/MSFC by Smithsonian Astrophysical Observatory.

[5] Beletsky, V. V. and Levin, E. M., Dynamics of Space Tether Systems, Vol. 83 of Advances in Astronautical Sciences, Univelt, Incorporated, P.O. Box 28130, San Diego, California 92198, 1993.

[6] Decou, A. B., "Tether Static Shape for Rotating Multimass, Multitether, Spacecraft for Triangle Michelson Interferometer," Journal of Guidance and Control, Vol. 12, No. 2, March-April 1989, pp. 273-275.

[7] Decou, A. B., "Attitude and Tether Vibration Control in Spinning Tethered Triangles for Orbiting Interferometry," The Journal of the Astronautical Sciences, Vol. 41, No. 3, JulySeptember 1993, pp. 373-398.

[8] Keshmiri, M. and Misra, A. K., "General Formulation for $\mathrm{N}$ body Tethered Satellite System Dynamics," Journal of Guidance, Control and Dynamics, Vol. 19, No. 1, JanuaryFebruary 1996, pp. 75-83.

[9] Misra, A. K., Bellerose, J., and Modi, V. J., "Dynamics of a Tethered System near the Earth-Moon Lagrangian Points," Proceedings of the 2001 AAS/AIAA Astrodynamics Specialist Conference, Quebec City, Canada, Vol. 109 of Advances in the Astronautical Sciences, 2002, pp. 415-435.

[10] Farquhar, R. W., "Tether Stabilization at a Collinear Libration Point," The Journal of the Astronautical Sciences, Vol. 49, No. 1, January-March 2001, pp. 91-106.

[11] Kim, M. and Hall, C. D., "Control of a Rotating VariableLength Tethered System," Proceedings of the 2003 AAS/AIAA Space Flight Mechanics Meeting, Ponce, Puerto Rico, Advances in the Astronautical Sciences, 2003 (to appear).

[12] Marino, R. and Tomei, P., "Global Adaptive Output-Feedback Control of Nonlinear Systems, Part I: Linear Parametrization," IEEE Transactions on Automatic Control, Vol. 38, No. 1, January 1993, pp. 17-32.

[13] Marino, R. and Tomei, P., "Global Adaptive Output-Feedback Control of Nonlinear Systems, Part II: Nonlinear Parametrization," IEEE Transactions on Automatic Control, Vol. 38, No. 1, January 1993, pp. 33-48. 
[14] Marino, R. and Tomei, P., “An Adaptive Output Feedback Control for a Class of Nonlinear Systems with Time-Varying Parameters," IEEE Transactions on Automatic Control, Vol. 44, No. 11, November 1999, pp. 2190-2194.

[15] Krsti'c, M. and Kokotovi'c, P., "Adaptive Nonlinear OutputFeedback Schemes With Marino-Tomei Controller," IEEE Transactions on Automatic Control, Vol. 41, No. 2, February 1996, pp. 274-280.

[16] Khalil, H. K., "Adaptive Output Feedback Control of Nonlinear Systems Represented by Input-Output Models," IEEE Transactions on Automatic Control, Vol. 41, No. 2, February 1996, pp. 177-188.

[17] Kim, M. and Hall, C. D., "Lyapunov and Halo Orbits about L2," Proceedings of the 2001 AAS/AIAA Astrodynamics Specialist Conference, Quebec City, Canada, Vol. 109 of Advances in the Astronautical Sciences, 2002, pp. 349-366.

[18] Farley, R. E. and Quinn, D. A., "Tethered Formation Configurations - Meeting the Scientific Objectives of Large Aperture and Interferometric Science," Proceedings of the AIAA Space 2001 - Conference and Exposition, Albuquerque, New Mexico, 2001.

[19] Gates, S. S., "Multi-tethered Space-based Interferometers: Particle System Model," Tech. Rep. NRL/MR/8231-01-8579, Naval Research Laboratory, Washington, DC 20375-5320, September 2001.

[20] Nicosia, S. and Tomei, P., "Robot Control by Using Only Joint Position Measurements," IEEE Transactions on Automatic Control, Vol. 35, No. 9, September 1990, pp. 1058-1061.

[21] Ohkami Y, Yoshimura S, Okamoto O. "Evaluation of microgravity level fluctuation due to attitude/orbital motion in a tethered satellite system," Acta Astronautica, Vol. 35 (2/3), 1995, pp. 187-91.

[22] Licata R. "Tethered system deployment controls by feedback fuzzy logic," Acta Astronautica, Vol. 40, No. 9, 1997, pp. 619-34.

[23] Nohmi M, Nenchev DN, "Uchiyama M. Momentum control of a tethered space robot through tether tension control," In: Proceedings of the IEEE international conference on robotics \& automation. IEEE, 1998, pp. 920-5.

[24] Woo MP, Misra AK. "Dynamics of tethered space manipulators," In: Proceedings of the AAS/AIAA astrodynamics specialist's conference, 2003, AAS [AAS 03534].

[25] Kumar K, Kumar KD. "Variable attitude maneuver through tether for a "drifting" twin satellite system in elliptic orbits," Acta Astronautica, Vol. 45, No. 3, 1999, pp. 135-42.

[26] Pradhan S, Modi VJ, Misra AK., "Tether-platform coupled control,"Acta Astronautica, Vol. 44, No. 5, 1999, pp. 243-56.

[27] Fujii HA, Taira W, Watanabe T, Murase T, Kusagaya T, Trivailo P., "Experimental analysis of deployment/retrieval of tether system using balloon technique," In: Proceedings of the AAS/AIAA astrodynamics specialists conference, 2001, AAS [AAS 01-407].

[28] Kumar KD, Yasaka T., "Satellite attitude stabilisation through kitelike tether configuration," Journal of Spacecraft and Rockets, Vol. 39, No. 5, 2002, pp. 755-60.
[29] Quadrelli MB. "Modelling and dynamics analysis of tethered formations for space interferometry," In: Proceedings of the AAS/ AIAA space flight mechanics meeting, 2001, AAS [AAS 01-231].

[30] Cho S, McClamroch NH., "Attitude control of a tethered spacecraft," In: Proceedings of the American control conference. IEEE, 2003, pp. 1104-9.

[31] Pela' ez J, Lara M., "Periodic solutions in electrodynamic tethers on inclined orbits," Journal of Guidance, Control and Dynamics, 2003, Vol. 26, No. 3, pp. 395-406.

[32] Rossi EV, Cicci DA, Cochran Jr JE., “ Existence of periodic motions of a tether trailing satellite," Appl Math Comput 2004; 155: 269-81.

[33] Bernelli-Zazzera F. Active control of tether satellites via boom rotation: a proof of concept experiment. In: Proceedings of THE AAS/AIAA space flight mechanics meeting, p. 1225-40 [AAS. 01- 192].

[34] Takeichi N, Natori MC, Okuizumi N, Higuchi K., " Periodic solutions and controls of tethered systems in elliptic orbits," $J$ Vib Control, 2004, Vol. 10, pp. 1393-413.

[35] Kumar K, Kumar KD., "Tethered dual spacecraft configuration: a solution to attitude control problems," Aerospace Sci Technol, 2000, Vol. 4, pp. 495-505.

[36] Djebli A, El-Bakkali L, Pascal M., "On fast retrieval laws for tethered satellite systems," Acta Astronautica, 2002, Vol. 50, No. 8, pp. 461-70.

[37] Hokamoto S, Imamura N, Modi VJ., "Dynamics and control of a tethered space robot with tension," In: Proceedings of the AAS/ AIAA astrodynamics specialists' conference, 2001, AAS [AAS 01- 408].

[38] Bombardelli C, Lorenzini EC, Quadrelli MB., "Retargeting dynamics of a linear tethered interferometer," $J$ Guidance Control Dynam, 2004, Vol. 27, No. 6, pp. 1061-7.

[39] Kumar, K. D., and Kumar, K., "Attitude Maneuver of Dual Tethered Satellite Platforms through Tether Offset Change," Journal of Spacecraft and Rockets, Vol. 38, No. 2, 2001, pp. 237-242.

[40] Beard R. W. and Hadaegh F. Y., "Finite thrust control for satellite formation flying with state constraints," American control conference, American Automatic control council, Sandiego, CA, 1999, pp. 2975-2979.

[41] Mori, O., and Matunaga, S., "Research and Development of Tethered Satellite Cluster Systems," International Conf. on Intelligent Robots and Systems, Paper F-AI-5-2, Oct. 2000.

[42] Moccia, A., Vetrella, S., and Grassi, M., "Attitude Dynamics and Control of aVertical Interferometric RadarTethered Altimeter," Journal of Guidance, Control, and Dynamics, Vol. 16, No. 2, 1993, pp. 264-269.

[43] Pradhan, S., Modi, V. J., and Misra, A. K., "On the Offset Control of Flexible Nonautonomous Tethered Two-Body Systems," Acta Astronautica, Vol. 38, No. 10, 1996, pp. 783801.

[44] Hokamoto, S., "Dynamics of a Space Robot with Tether Tension," Proceedings of 10thWorkshop on Astrodynamics and Flight Mechanics, Inst. of Space and Astronautical Science, Sagamihara, Japan, 2000, pp. 237-242. 
[45] Matunaga, S., Ohkami, Y., and Mori, O., "A Tether-Based Capture of Orbiting Objects," International Astronautical Congress, Paper 97-A.3.08, Oct. 1997.

[46] Matunaga, S., Mori, O., Nakaya, K., and Ohkami, Y., "Tether Control Methods for Damping Angular Momentum of Uncontrolled Satellites," Proceedings of 8th Workshop on Astrodynamics and Flight Mechanics, Institute of Space and Astronautical Science, Sagamihara, Japan, 1998, pp. 310-315.

[47] Kumar, K. D., and Nakajima, A., "Angular Momentum Damping of Debris Through Tether System," International Symposium on Space Technology and Science, Paper 2000-k20, May 2000.

[48] Nohmi, M., Terumichi, Y., and Sogabe, K., "Modeling of Tethered Space Robot as String-Rigid Bodies," Proceedings of 10th Workshop on Astrodynamics and Flight Mechanics, Inst. of Space and Astronautical Science, Sagamihara, Japan, 2000, pp. 243-248.

[49] Wong, B., and Misra, A. K., "Dynamics of a Tethered System near the Earth-Moon Lagrangian Points," Proceedings of the 2003 AAS/AIAA Space Flight Mechanics Meeting, Vol. 114, Advances in the Astronautical Sciences, Univelt Inc. Publishers, San Diego, CA, 2003, pp. 1675-1694.

[50] Loria, A., "Global Tracking Control of One Degree of Freedom Euler- Larange Systems with Velocity Measurements," European Journal of Control, Vol. 2, No. 2, June 1996, pp. 144-151.

[51] de Queiroz, M. S., Dawson, D. M., Nagarkatti, S. P., and Zhang, F., Lyapunov-Based Control of Mechanical Systems, Control Engineering, Birkhäuser Boston, Cambridge, MA, 2000 .

[52] Isidori, A., Nonlinear Control Systems, 3rd ed., SpringerVerlag, New York, 1995, Chap. 5.

[53] Kojima, H., Iwasaki, M., Fujii, A. H., Blanksby, C., and Trivailo, P., "Nonlinear Control of librational Motion of Tethered Satellites in Elliptic Orbits," Journal of Guidance, Control, and Dynamics, Vol. 27, No. 2, 2004, pp. 229-239.

[54] Kojima H. and Sugimoto T., "Nonlinear control of double pendulum Electrodynamic tether system" journal of space craft and rockets, Vol. 44, No. 1, 2007 DOI: 10.2514/1.24537.

[55] Corsi J. and Less L., "Stabillity and control of electrodynamics tethers for deorbiting applications," Acta Astronautica, Vol. 48, No. 5-12, 2001, pp. 491-501.

[56] Tortora P, Somenzi L., Less 1., and Licata R., "Small mission design for testing in orbit an electrodynamic tether deorbiting system," Journal of spacecraft and Rockets, Vol. 43, No. 4, 2006, pp. 883-892. Doi: 10.2514/1.15359.

[57] Sun F. J., Zhu Z. H. and La Rosa M., "Dynamic modelling of cable towed body using nodal positon finite element method," Ocean Engineering, Vol. 38, No. 4, 2011, pp. 529-540.

[58] Gangqiang Li, Zheng H., Zhu, Czekanski A., "Libration control of bare electrodynamic tethers considering elasticthermal-electrical coupling," Journal of guidance, control and Dynamics, Vol. 39. No. 3, 2016.

[59] Manchester Z. and Peck M. A., "Stochastic space exploration with microscale spacecraft," AIAA, Guidance, navigation and control conference, AIAA paper 2011-6648, 2011, pp. 8-11.
[60] Weis L. M. and Peck M. A., "Attitude control for chip satellites using multiple electrodynamic tethers," AIAA/AAS Astrodynamic specialist conference, AIAA paper 2012-4871, 2012. Doi: 10.2514/6.2012-4871.

[61] Lorraine M. W. and Mason P., "Chip-scale satellite control with multiple electrodynamic tethers," Journal of guidance, control and dynamics, Vol. 39, No. 7, 2016.

[62] S. V. Drakunov and V. I. Utkin, " Sliding mode control in dynamic systems" international journal of control, Vol. 55, No. 4, pp. 1029-1037, 1992.

[63] J. J. E. Slotine and Sastry, "Sliding control design for nonlinear systems," Vol. 40 No. 2, 1984, pp. 421-434.

[64] Hallaj, M. A. A. and Assadian N.," Sliding mode control of electrodynamic tethered satellite formation", Advances in space research. Vol. 58, No. 4, 2016, pp. 619-634.

[65] Walls, J., and Greene, M., "Adaptive Control of an Orbiting Single Tether System," Proceedings of the Twenty-First Southeastern Symposium on Systems Theory, Inst. of Electrical and Electronics Engineers, Piscataway, NJ, Mar. 1989, pp. 594-598.

[66] Bartolini, G., Levant, A., Plestan, F., Taleb, M., \& Punta, E. (2013), "Adaptation of sliding modes," IMA Journal of Mathematical Control and Information, 30 (3), 285-300.

[67] Edwards, C., \& Shtessel, Y. (2016) "Adaptive continuous higher order sliding mode control," Automatica, 65, 183-190.

[68] Edwards, C., \& Spurgeon, S. (1998). Sliding mode control: Theory and applications, London: Taylor and Francis.

[69] Q. Hu, "Robust adaptive sliding mode attitude maneuvering and vibration damping of three-axis-stabilized flexible spacecraft with actuator saturation limits" Nonlinear Dynamics, 55 (4), 301-321, 2009.

[70] Boskavic, J. D., Li, S. M., Mehra R. K., “ Robust adaptive variable structure control of spacecraft under control input saturation" Journal of guid, control and dyn. Vol. 24. No. 1, pp 14-22 2001.

[71] Ma Zqiang and Sun Guanghui, "Adaptive sliding mode control of tethered satellite deployment with input limitation," Acta Astronautica, vol. 127, pp. 67-75, 2016.

[72] Manabe, S., "The non -integer integral and its application to controll systems," Electrotechnical Journal of Japan, 6 (3-4), 83-87.

[73] Sun, G. and Zhu, Z. H., "Fractional order tension control for stable and fast tethered satellite retrieval," Acta astronautica, vol. 104. No. 1 pp. 304-312 2014.

[74] Pradup S. "Anew tension control law for deployment of tethered satellites," Mechanics Research communication, Vol. 24, No. 3, 1997, pp. 247-254.

[75] Kang J, Zheng, H. Zhu, Wang W., Li A., Wang C. "Fractional order sliding mode control of tethered satellite deployment with disturbances", Advance in space research, vol. 59. No., 2017, pp. 263-273.

[76] Venkataraman, S., Gulati, S., "Control of Nonlinear Systems Using Terminal Sliding Modes," J. Dyn. Sys, Meas., Control, Sept 1993, Volume 115, Issue 3.

[77] Yang and Yang. 
[78] B. S. Yu, D. P. Jin and H. Wen, "A method of stable deployment of electrodynamic tethered satellite in three dimensional space," journal of physics, Vol. Conference Series 744 (2016) 012119.

[79] Kojima H. and Sugimoto T., "Stability analysis of in-plane and out-plane periodic motions to electrodynamic tether system in enclined elliptic orbit," Acta Astronautica, Vol. 65, No. 3-4, 2009, pp. 477-488.

[80] J. Palaez and E. C. Lorenzini, "Libration control of electrodynamic tethers in confined orbit," Journal of Guidance, Control and Dynamics, Vol. 28, 2005, pp. 269-279.

[81] M. Inarrea and J. Palaez, "Libration control of electrodynamic tether using the ETDAS method," Journal of Guidance, control and Dynamics, Vol. 33, 2010, pp. 923-933.

[82] V. Lanchares, M. Inarrea, A. I. Pascuel, J. P. Salas, “Attitude stabilization of electrodynamic tethers in elliptic orbits by time delayed feedback control," Acta Astronautica, Vol. 96, 2014, pp. 280-295.

[83] Williams, P.: "Optimal deployment/retrieval of a tethered formation spinning in the orbital plane," Journal of Spacecraft and Rockets, Vol. 43 (3), pp. 638-650, (2006).

[84] Williams P., " Optimal control of electrodynamic tether orbit transfer using time scale separation," Journal of Guidance, control and Dynynamics, Vol. 33, No. 1, 2010, pp. 88-99.
[85] Misra A. K and Modi V. S., " Deployment and retrieval of shuttle supported tethered satellites" Journal of Guidance, Control and Dynamics, Vol. 5, No. 3, 1982, pp. 278-285.

[86] Dongpin J and Haiyan H., “ Optimal control of tethered sub satellite of three degrees of freedom" Nonlinear dynamics, Vol. 46, No. 1, 2006, pp. 161-178.

[87] Zhang R. and Zhu Z. H., "Optimal control of nanosatellite fast deorbit using electrodynamic tethers" Journal of Guid., Cont and Dyn, Vol. 37, No. 4, 2014, pp. 1182-1194.

[88] Williams, P., "Optimal control of a spinning double-pyramid earth pointing tether formation," In: The 57th International Astronautical Congress, Valencia, 2-6 October (2006).

[89] Bainum, P. M and Kumar V. K., "Optimal control of electrodynamic tethers for deorbiting applications," Acta Astronautica, Vol. 7, No. 12, 1980, pp. 1333-1348.

[90] Godard K. D, Kumar and B. Tan, " nonlinear optimal control of tethered satellite system using tether offset in presence of tether failure" Acta Astronautica, Vol. 66, No., 2010, pp. 1434-1448. 